\title{
Comparative genomics and experimental promoter analysis reveal functional liver-specific elements in mammalian hepatic lipase
}

\section{genes}

\author{
Diederik van Deursen ${ }^{1}$, Gert-Jan Botma1, Hans Jansen ${ }^{1,2}$ and \\ Adrie JM Verhoeven*1
}

Address: ${ }^{1}$ Department of Biochemistry, Cardiovascular Research School COEUR, Erasmus MC, PO Box 1738, 3000 DR Rotterdam, The Netherlands and ${ }^{2}$ Department of Clinical Chemistry, Cardiovascular Research School COEUR, Erasmus MC, PO Box 1738, 3000 DR Rotterdam, The Netherlands

Email: Diederik van Deursen - d.vandeursen@erasmusmc.nl; Gert-Jan Botma - GJBotma@prdgb.JNJ.com;

Hans Jansen - h.jansen@erasmusmc.nl; Adrie JM Verhoeven* - a.verhoeven@erasmusmc.nl

* Corresponding author

Published: II April 2007

BMC Genomics 2007, 8:99 doi:10.1 186/1471-2164-8-99

This article is available from: http://www.biomedcentral.com/I47/-2164/8/99

(C) 2007 van Deursen et al; licensee BioMed Central Ltd.

This is an Open Access article distributed under the terms of the Creative Commons Attribution License (http://creativecommons.org/licenses/by/2.0), which permits unrestricted use, distribution, and reproduction in any medium, provided the original work is properly cited.
Received: 23 January 2007

Accepted: II April 2007

\begin{abstract}
Background: Mammalian hepatic lipase $(\mathrm{HL})$ genes are transcribed almost exclusively in hepatocytes. The basis for this liver-restricted expression is not completely understood. We hypothesized that the responsible cis-acting elements are conserved among mammalian $\mathrm{HL}$ genes. To identify these elements, we made a genomic comparison of $30 \mathrm{~kb}$ of 5 -flanking region of the rat, mouse, rhesus monkey, and human HL genes. The in silico data were verified by promoterreporter assays in transfected hepatoma HepG2 and non-hepatoma HeLa cells using serial 5'deletions of the rat $\mathrm{HL}(-2287 /+9)$ and human $\mathrm{HL}(-685 /+13)$ promoter region.

Results: Highly conserved elements were present at the proximal promoter region, and at 14 and $22 \mathrm{~kb}$ upstream of the transcriptional start site. Both of these upstream elements increased transcriptional activity of the human $\mathrm{HL}(-685 /+13)$ promoter region $2-3$ fold. Within the proximal $\mathrm{HL}$ promoter region, conserved clusters of transcription factor binding sites (TFBS) were identified at $-240 /-200$ (module A), $-80 /-40$ (module B), and $-25 /+5$ (module C) by the rVista software. In HepG2 cells, modules $B$ and $C$, but not module $A$, were important for basal transcription. Module $B$ contains putative binding sites for hepatocyte nuclear factors HNFI $\alpha$. In the presence of module B, transcription from the minimal HL promoter was increased I.5-2 fold in HepG2 cells, but inhibited 2-4 fold in HeLa cells.
\end{abstract}

Conclusion: Our data demonstrate that searching for conserved non-coding sequences by comparative genomics is a valuable tool in identifying candidate enhancer elements. With this approach, we found two putative enhancer elements in the far upstream region of the $\mathrm{HL}$ gene. In addition, we obtained evidence that the $-80 /-40$ region of the $\mathrm{HL}$ gene is responsible for enhanced $\mathrm{HL}$ promoter activity in hepatoma cells, and for silencing $\mathrm{HL}$ promoter activity in non-liver cells. 


\section{Background}

Understanding transcriptional regulation of gene expression is a major challenge in molecular biology. In eukaryotes, regulation of gene expression is achieved through the complex interaction of transcription factors, which bind to specific DNA sequence motifs. These motifs are predominantly located in the upstream region of genes. Over the last decades, numerous transcription factors have been identified, each with its own specific DNA binding sequence (TFBS). Transcription factors that are potentially involved in the regulation of a particular gene are usually identified by the presence of the specific DNA binding motif in the upstream regulatory region. These binding motifs are compiled in libraries such as the Transfac database [1], and programs such as MatInspector enable pattern recognition with the entries in this database [2]. Unfortunately, most transcription factors bind to short, degenerate sequences, which occur very frequently in the eukaryotic genome. Only a very small fraction of all predicted binding sites is biologically relevant [3]. Recently, new strategies for the $a b$ initio identification of functionally significant $c i s$-acting regulatory sequences have been developed, based on the assumption that regulatory elements are conserved among multiple species [4-8], and that multiple TFBS tend to specifically cluster together $[9,10]$. The rVista computational tool for identification of functional regulatory elements combines the comparative sequence analysis of orthologous genes with the analysis of clustering of predicted TFBS [11,12]. In this study, we tested the validity of this approach to identify functional TFBS for the mammalian hepatic lipase genes, by comparing the in silico data with experimental promoter-reporter assays.

Hepatic lipases (HL) are synthesized and secreted almost exclusively by hepatocytes [13-15]. Although synthesis of HL has been shown to occur in mouse adrenals [16], and in mouse and human macrophages [17], this is negligible compared to expression in liver. The HL activity present in adrenals and ovaries [18] originates predominantly from liver, and is transported through the circulation to these organs $[19,20]$. In liver, the enzyme is bound to cell surface proteoglycans within the sinusoids, from where it can be released by heparin. Hepatic lipase plays an important role in plasma lipoprotein metabolism and intracellular lipid homeostasis [21], by mediating cholesterol influx into liver cells from high-density lipoproteins (HDL), and clearance of remnant lipoproteins from the circulation by the liver. HL is an important determinant of plasma HDL cholesterol levels, and is implicated in the protection against development of premature atherosclerosis by HDL [21]. HL gene expression in humans and rodents is regulated by various hormones and nutritional states mainly at the transcriptional level, but up- or downregulation is limited to about two-fold [15]. In contrast to this moder- ate regulation by hormones and nutrition, the almost complete restriction of HL gene expression to differentiated liver cells is highly conspicuous $[13,14]$. Several groups have pointed to the HNF1 and HNF4 $\alpha$ binding sites in the proximal promoter of the HL gene to explain this liver-specificity in humans [22-25]. Since the liverrestricted expression is a common feature of most, if not all, mammalian HL genes, we hypothesize that the regulatory elements responsible for liver-specific expression are conserved among mammals. We therefore searched the upstream regulatory region of the rat, mouse, rhesus monkey and human genes for the presence of conserved clusters of TFBS motifs, and combined the in silico data with experimental promoter-reporter assays in cultured cells of hepatic versus non-hepatic origin. This unbiased approach led to the identification of two putative enhancer elements in the far upstream region, and of highly conserved sequence modules within the proximal promoter of the HL genes.

\section{Results}

\section{Interspecies comparison of genomic $H L$ sequences}

Of the mammalian HL genes, genome sequence including part of the 5'-flanking region is available for human, chimpanzee, rhesus monkey, rat, mouse and hedgehog (Ensembl $\boldsymbol{e}$ !42:Dec 2006)[26]. Pairwise alignment of the HL coding sequences shows the expected, high degree of sequence identity (Table 1). This high homology also extends into the 5'-UTR and upstream-regulatory region, with sequence identity ranging from 53 to $98 \%$ over the proximal 0.9-1.4 kb. Multiple sequence alignment of 30$\mathrm{kb}$ of the 5 '-flanking region available for five HL genes (all except for the hedgehog, which known sequence is too short) was performed by the mVista web-tool (Fig. 1). The chimp and macaque sequences are highly homologous to the human sequence, as $95 \%$ and $64 \%$ of the $30-\mathrm{kb}$ region showed at least $95 \%$ sequence identity over a 100bp window, respectively. Similarly, $5 \%$ of the rat sequence showed this high degree of sequence identity with the orthologous mouse sequence. Because of the near-identity of the chimp to the human sequence, we only included the latter sequence in subsequent in silico analysis. The global genomic sequence comparison showed a particularly high conservation among the four genes immediately upstream of the transcriptional start site $\left(P=7^{*} 10^{-7}\right)$. Three additional islands of highly significant homology $\left(P<10^{-5}\right)$ were identified further upstream. Conservation of a $475 \mathrm{bp}$ element at $-14 \mathrm{~kb}$ was even more significant $\left(P=4^{*} 10^{-11}\right)$ than the proximal HL promoter region (Fig. 1). The element at $-22 \mathrm{~kb}\left(P=2 * 10^{-}\right.$ $\left.{ }^{6}\right)$ contained a 173 bp sequence that was completely identical among the three primate sequences.

To test the potential enhancer function of two of the most conserved elements in the far upstream regulatory region, 
Table I: Pairwise sequence comparison of the coding and the 5 '-flanking sequence of mammalian HL genes.

\begin{tabular}{|c|c|c|c|c|c|c|c|c|}
\hline \multicolumn{9}{|c|}{ upstream regulatory sequence } \\
\hline \multirow{7}{*}{$\begin{array}{l}\text { cDNA } \\
\text { sequence }\end{array}$} & 81 & & 53 & 53 & 61 & 57 & 56 & hedgehoga \\
\hline & 79 & 76 & & 86 & 64 & 55 & 59 & mouse \\
\hline & 79 & 76 & 92 & & 63 & 57 & 57 & rat \\
\hline & 85 & 82 & 79 & 79 & & 94 & 94 & rhesus $^{\mathrm{a}}$ \\
\hline & 85 & 82 & 80 & 80 & 96 & & 98 & chimp \\
\hline & 85 & 82 & 79 & 79 & 96 & 99 & & human \\
\hline & rabbit ${ }^{b}$ & hedgehog & mouse & rat & rhesus & chimp & human & \\
\hline
\end{tabular}

Sequence identity (\%) was determined by pairwise alignment using the DNAMAN software package (optimal alignment; gap open penalty 10.0, gap extension penalty 5.0). cDNA sequence was from the translation start ATG up till the stopcodon; the upstream regulatory region was from - 1400 up till the translation start ATG.

a: Because of a gap in upstream regulatory region of the public sequence of the rhesus monkey and hedgehog HL gene (Ensembl e!42: Dec 2006), alignments with the rhesus and hedgehog sequence were done with the sequence from -900 and -1000 up till the start ATG, respectively.

b:the rabbit HL cDNA sequence was taken from [38].

promoter-reporter assays were performed with human HL promoter constructs in transiently transfected HepG2 hepatoma cells. The $-14 \mathrm{~kb}$ element ( $679 \mathrm{bp}$ ) and the -22 $\mathrm{kb}$ element (387 bp) were inserted in the hHL-685Luc plasmid. As a control, we also tested the activity of the non-conserved -10 kb sequence (502 bp). The transcriptional activity of the human HL $-685 /+13$ promoter region was increased 3 - and 2 -fold with the $-14 \mathrm{~kb}$ and $22 \mathrm{~kb}$ elements inserted in the sense orientation, respectively (Fig. 2). In contrast, the $-10 \mathrm{~kb}$ sequence slightly but significantly reduced HL promoter activity $(\mathrm{n}=3 ; \mathrm{P}<$ 0.05). Qualitatively similar effects were obtained when the elements were inserted in the anti-sense orientation (Fig. 2). Hence, the conserved sequences at $-14 \mathrm{~kb}$ and -22 $\mathrm{kb}$ have moderate enhancer activity of the proximal HL promoter region.

\section{Comparative genomics of the proximal $2 \mathrm{~kb} H \mathrm{H}$ upstream regulatory region}

Submission of 2-kb upstream sequence of the rat HL gene to the MatInspector software program (core similarity > 0.75 ; matrix similarity $>0.70$ ) returned over 2000 potential TFBS, randomly distributed over the entire sequence. A similar number of sites was predicted for the orthologous mouse, human and rhesus macaque sequences. When we searched for clustered TFBS motifs that are conserved between the rat and human sequence, using the web-tool rVista, three separate modules were identified within the proximal promoter region (Fig. 3). Module A (240 to -200 in the human sequence relative to the transcriptional start site), for which AP1, AP2, CAAT, COUP, $\mathrm{C} / \mathrm{EBP}, \mathrm{HNF} 4 \alpha, \mathrm{PPAR}$ and USF binding sites are predicted, corresponds to the DR1 site recently identified by Rufibach et al. [25]. Module B (-80 to -40$)$ potentially contains AP2, CAAT, C/EBP, HNF1, HNF4, PPAR and Sp1 sites, and corresponds to the previously characterized HNF1 site [22-24]. Module C $(-25$ to +5$)$, which may bind AP2, C/EPB, HNF4, PPAR and USF factors, contains the transcription start site preceded by a conserved pyrimidine-rich motif, and therefore likely represents the Inr involved in binding of the transcription initiation complex. These three modules were also found to be conserved among the human and mouse HL gene. The human-mouse comparison revealed an additional, conserved module (-295 to -265), with potential binding sites for AP2, C/EBP, HNF1, HNF4, PPAR and Sp1, and which partly overlaps the DR4-site recently described by Rufibach et al. [25]. Similar results were obtained in pairwise comparisons between orthologous sequences of macaque and rat, and of macaque and mouse. Despite the high homology in the intervening sequence between modules $\mathrm{A}$ and $\mathrm{B}$, the rVista program did not recognize conserved clusters of TFBS among the human, macaque, rat and mouse. Irrespective of which transcription factors actually bind to these sites, the results of the interspecies sequence comparison by rVista suggest that the three highly conserved sequence modules in the proximal HL promoter region are involved in common features of transcriptional regulation. This is further supported by the fact that these three modules correspond to distinct DNA footprints of the human HL sequence in rat liver [22] and human HepG2 cells [23].

\section{Functional characterization of the rat $\boldsymbol{H L}$ promoter region} To corroborate the in silico results, promoter-reporter assays were performed with promoter fragments of the rat HL gene in transiently transfected HepG2 cells. Plasmids were constructed with progressively 5 '-deleted promoter fragments spanning the $-2287 /+9$ region of the rat $\mathrm{HL}$ gene in front of the CAT reporter gene. Compared to the SV40 promoter, the rHL-2287 construct showed low CAT expression (Fig. 4). Upon deleting the 5'-end of the HL promoter fragments to position -1048, CAT expression became even lower, and was no longer significantly differ- 


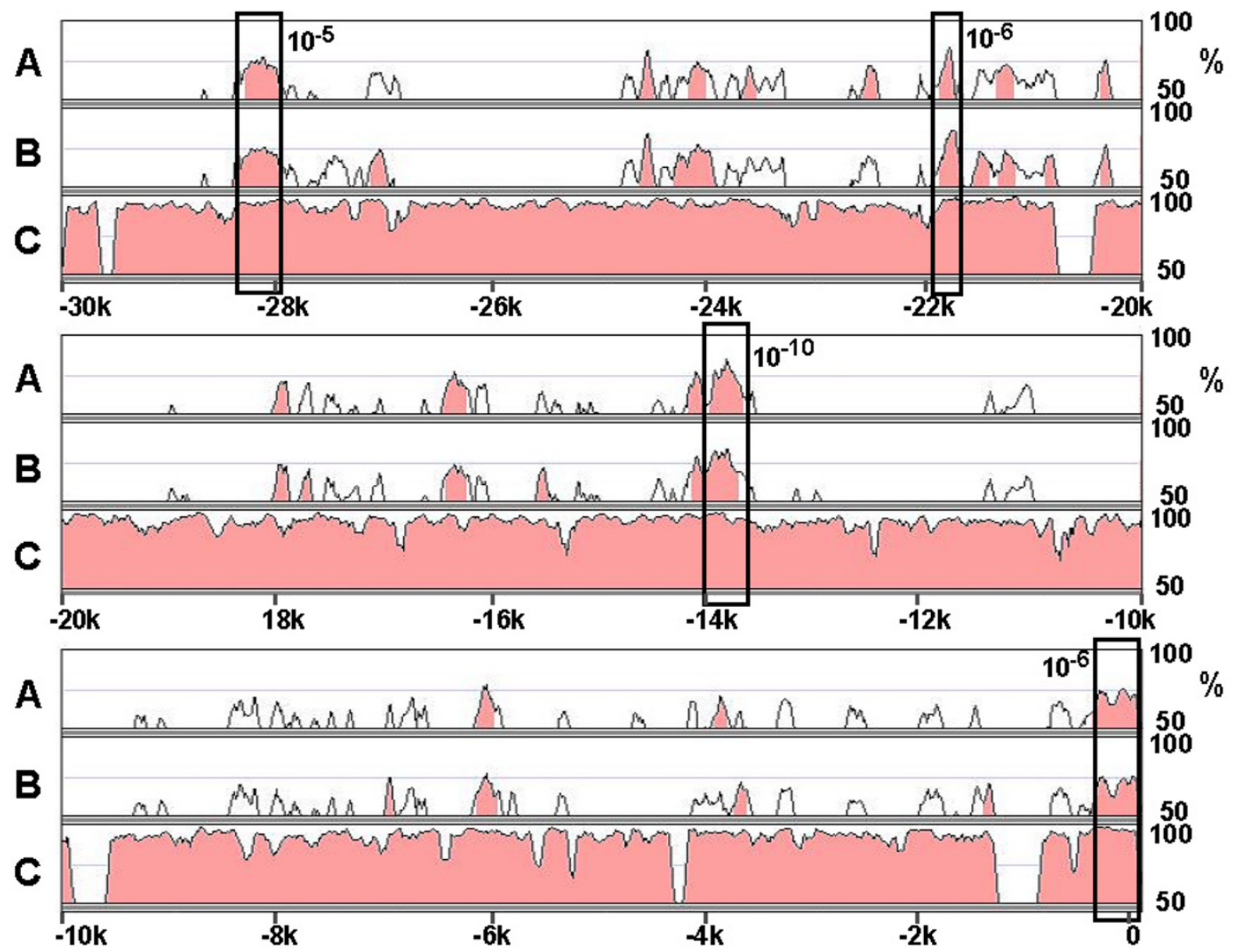

\section{Figure I}

Alignment of the 5'-flanking region of four mammalian $\mathbf{H L}$ genes . From the rat, mouse, macaque and human $\mathrm{HL}$ genes, exon- $\mathrm{I}$ and $30 \mathrm{~kb}$ of upstream sequence was aligned by the MLAGAN algorithm of the mVista program. The sequences of rat (A), mouse (B) and macaque $(C)$ are aligned to the human $H L$ sequence ( $x$-axis); numbering is relative to the transcriptional start site. Conserved regions (> 70\% homology over $100 \mathrm{bp}$ window) are shaded. The boxes indicate conserved regions among the four sequences, as determined by RankVista $\left(P \leq 10^{-5}\right)$, with the $P$-values given above.

ent from promoter-less pCAT-Basic, suggesting that there is weak enhancer activity between nucleotides -1697 and -2287. Further deletion to position -754 slightly increased promoter activity to levels significantly above background. Shortening the insert from -754 to -446 resulted in a 5 -fold increase in promoter activity, suggesting the presence of a strong negative regulatory element in this region of the rat HL gene. CAT expression was not significantly affected by deleting the insert from -446 to -211 . The presence of the weak enhancer element between 2287 and -1697 , and the negative element between -754 and -446 corresponds to positive and negative elements in the human HL upstream regulatory region observed by Oka et al. [23]. We assume, therefore, that both these elements are present in homologous parts of the rat and human gene. Indeed, the global alignment of the four species by mVista detected homology at these parts of the gene (Fig. 1), but homology did not exceed the $70 \%$ over 100 bp mark used as threshold in this analysis. Apparently, potentially important elements may be missed due to the high stringency of the conservation rule in the mVista program.

To test the importance of the conserved sequence modules within the -220 to +9 region, further 5 -deletions in the rat HL promoter region were made (Fig. 5). Transcriptional activity of the rHL-127 construct, in which module A has been removed, was not significantly different from that of the rHL-446 or rHL-221 constructs. Similarly, removal of the highly conserved intervening sequence 

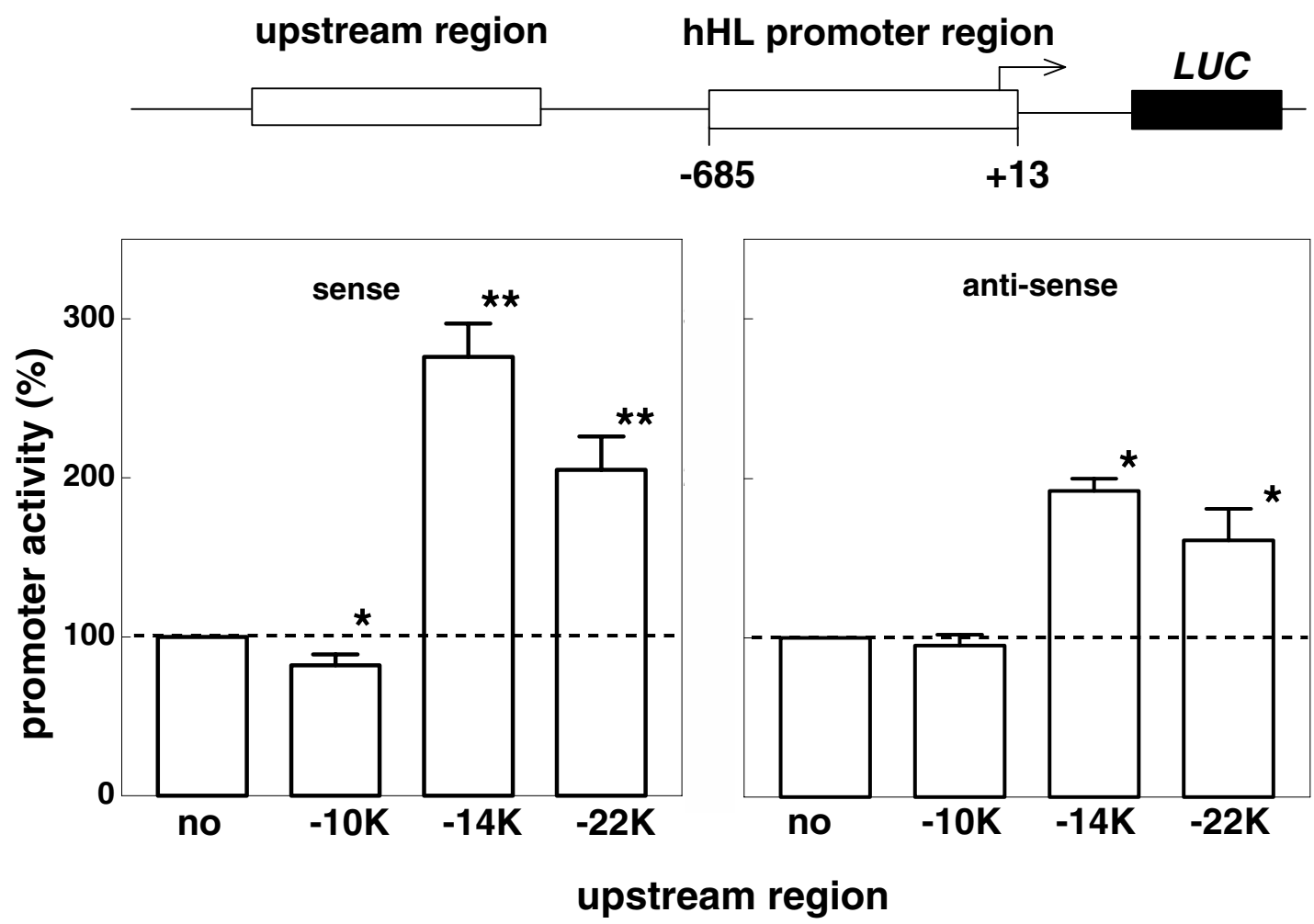

Figure 2

Possible enhancer activity of the conserved sequences in the far upstream HL regulatory region. HepG 2 cells were transiently transfected with the indicated promoter-reporter constructs. At $48 \mathrm{~h}$ post-transfection, transcriptional activity was determined as the firefly over renilla luciferase activity. Data are expressed as percentage of the ratio measured in the hHL-685luc - transfected cells. Data are means \pm SD from 3-4 independent experiments, each performed in quadruplicate. *and **: $P<0.05$; and $P<0.01$, respectively.

between modules A and B (rHL-86 and rHL-75) had no significant effect on CAT expression. In contrast, additional removal of most of module $\mathrm{B}$ in rHL-39 reduced transcriptional activity by approximately $60 \%$. With rHL23, in which the remainder of module $\mathrm{B}$ as well as the putative TATA-box has been deleted, CAT expression decreased further. Despite absence of the TATA-box, CAT expression of the rHL-23 construct was significantly higher than of promoter-less pCAT-Basic, which may be due to residual promoter activity of module $\mathrm{C}$.

\section{Comparison with the proximal human $H L$ promoter region} Similar promoter-reporter assays were performed with the $-685 /+13$ region of the orthologous human HL gene, except that the luciferase gene was used as reporter (Fig. $6)$. Luciferase activity of the hHL-306 construct was similar to hHL-685, whereas activity of the hHL-79 construct was slightly, but not significantly, higher. This is in line with the rat promoter data, which show little effect of module $\mathrm{A}$, and of the intervening sequence between modules A and B, on basal transcriptional activity in HepG2 cells. The luciferase activity of the hHL-36 construct, in which entire module B has been removed, was only $25 \%$ of the hHL-79 construct. The transcriptional activity of hHL-36, which contains a bona fide TATA box and entire module $\mathrm{C}$, was 7 -fold higher than background. This confirms that modules $\mathrm{B}$ and $\mathrm{C}$ are crucial for basal transcriptional activity in HepG 2 cells, with module B being most important.

\section{Role of module B in liver cell-specific HL transcription}

To test whether modules A and B are involved in liver-specific expression of the HL gene, we compared transcriptional activity of different rat HL promoter fragments in 


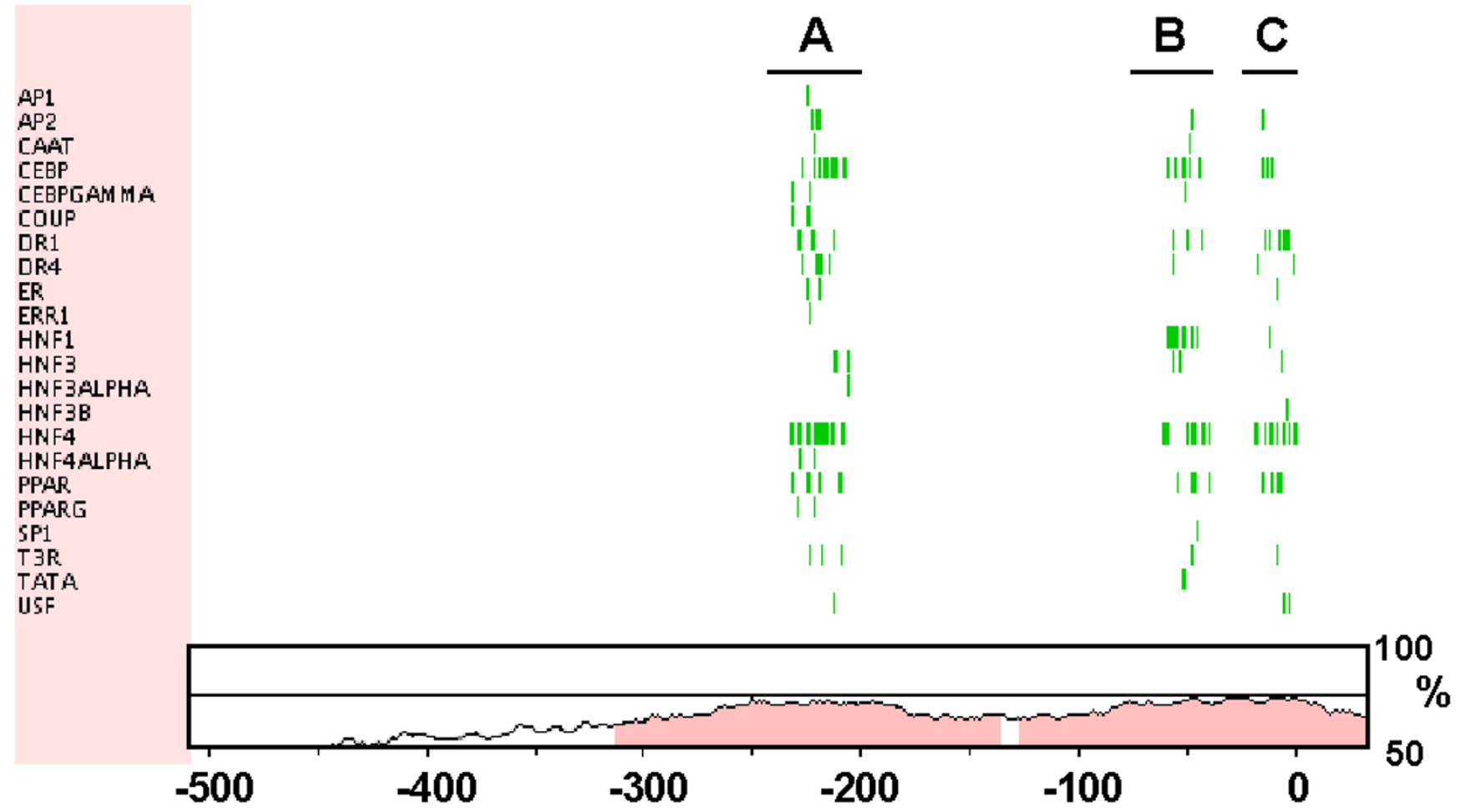

Figure 3

Identification of functional regulatory sequences in the proximal promoter region of the $\mathrm{HL}$ gene by $\mathbf{r V i s t a}$. Of the proximal promoter regions of the rat and human $\mathrm{HL}$ genes, 600 bp were submitted to the rVista sequence analysis software, and searched for conserved clusters of TFBS for a selection of 50 transcription factors known to be expressed in mammalian liver. A vertical line indicates the position of the conserved TFBS relative to the human sequence ( $x$-axis, numbering is relative to the transcriptional start site). Homology between the rat and human sequence is given as described for figure $\mathrm{I}$. Three clusters of conserved TFBS are identified, and designated A (-240/-200), B (-80/-40) and C (-25/+5). In human-mouse comparison, these clusters are also evident with an additional cluster at $-295 /-265$.

HepG2 cells with non-hepatic HeLa cells (Fig. 7). Promoter activity in each cell line was expressed as percentage of that of the rHL-39 construct, because this fragment represents the minimal promoter with the TATA-box and transcription start site. In the hepatoma cells, the activity of the rHL-75 construct was 1.5-2 fold higher than the minimal promoter construct. In HeLa cells, contrastingly, transcriptional activity of rHL-75 was 2-4 fold lower than the minimal promoter construct in HeLa cells. Consequently, there was a marked, 3-5-fold difference in relative promoter activity between these two cell lines. Similar results were obtained with the longer rat HL constructs that all contained module $\mathrm{B}$. The data were minimally affected by the simultaneous presence of module A (Fig. 7). Qualitatively similar results were obtained with human HL promoter fragments (data not shown). We conclude therefore, that module $\mathrm{B}$ plays a pivotal role in liver-restricted expression of the HL gene, by moderately activating transcription in liver cells, and simultaneously suppressing activity in non-hepatic cells.

\section{Discussion}

Global alignment of the 5'-flanking region of mammalian HL genes revealed three highly conserved elements $(\mathrm{P} \leq$ $10^{-5}$ ) that lie far upstream of the HL promoter (Fig. 1). Two of these elements, at $-14 \mathrm{~kb}$ and $-22 \mathrm{~kb}$, show moder- 


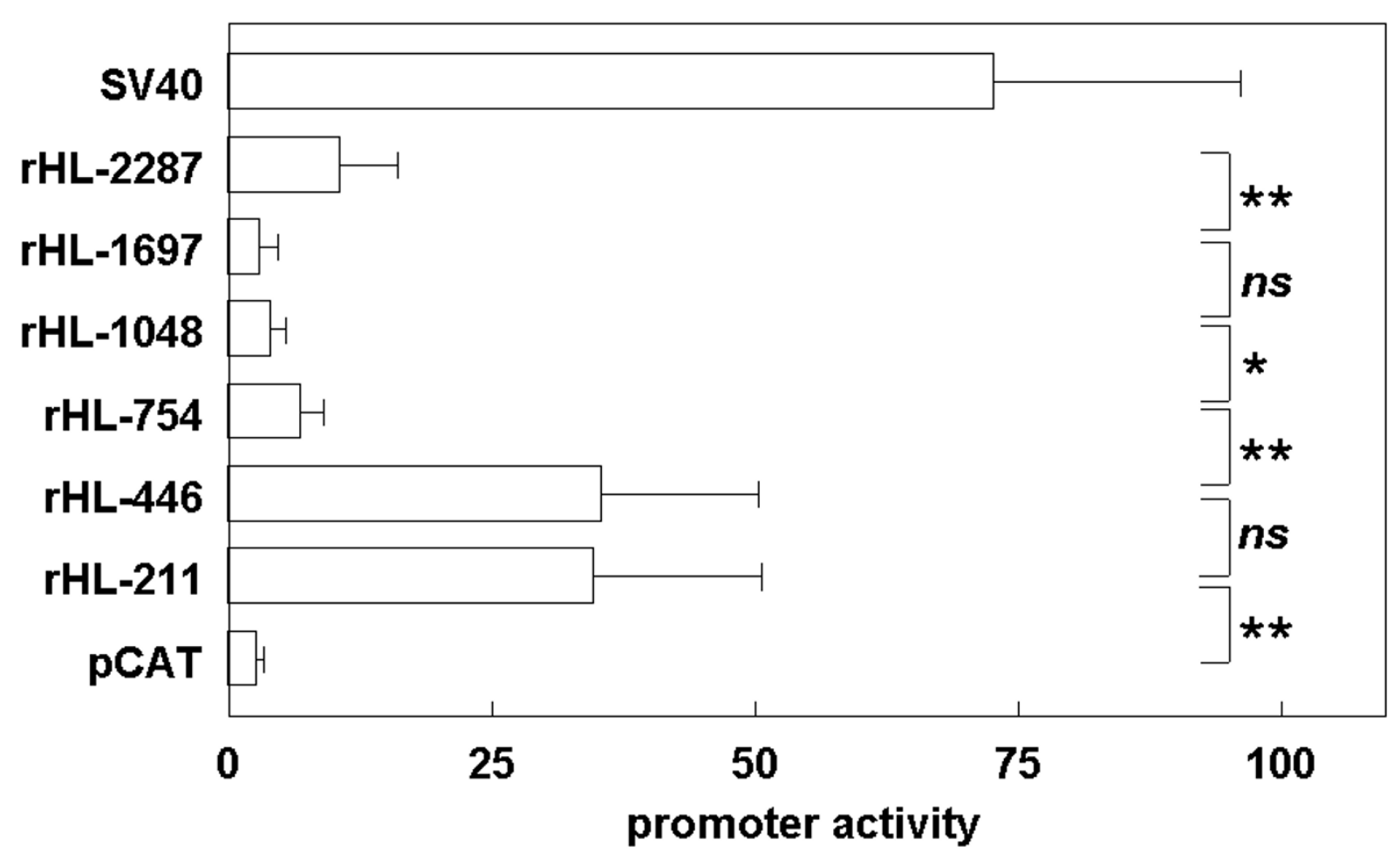

\section{Figure 4}

Effect of serial 5 '-deletions of the rat HL upstream region on transcriptional activity in HepG2 cells. HepG2 cells were transiently transfected with the indicated promoter-reporter constructs. At $48 \mathrm{~h}$ post-transfection, cells were lysed and expression of CAT and $\beta$-galactosidase protein was determined. Data are expressed as the ratio of CAT over $\beta$-galactosidase expression. Data are means \pm SD from 4-7 independent experiments, each performed in triplicate. $*: P<0.05$; $* *$ : $P<0.0$ I; n.s.: not significant.

ate enhancer activity in HepG2 cells. What discriminates the conserved $-14 \mathrm{~kb}$ and $-22 \mathrm{~kb}$ elements from the nonfunctional, non-conserved $-10 \mathrm{~kb}$ sequence is unclear, as all three sequences contain a similar repertoire of TFBS for liver-expressed transcription factors (data not shown). Further studies are required to clarify the mechanism responsible for the enhancer activity of the two highly conserved elements in the HL gene. The finding of two hitherto unknown enhancers supports the hypothesis that conserved non-coding sequences may identify functional regulatory elements. Experimentally, we also found a positive and a negative regulatory sequence between -2.2 and $-0.4 \mathrm{~kb}$ of the rat HL gene that coincided with homology peaks, but were not recognized by the Rankvista analysis of the sequence comparison. Rubin's group recently dem- onstrated strong in vivo enhancer activity for almost half of the elements that are ultra-conserved among human/ mouse/rat $[8,27]$. Our study further illustrates the power of the approach, and suggests that gene regulatory functions may also reside in somewhat less conserved elements among mammalian genomes.

We also tested whether global genome comparisons can also aid in identification of functional regulatory elements within highly conserved sequences, using the proximal HL promoter region as a model. Within this proximal promoter region, three modules are identified with conserved clusters of TFBS motifs. These modules A, $\mathrm{B}$ and $\mathrm{C}$ correspond with the previously identified regulatory elements DR1 [25], HNF1 [22-24] and Inr [22-24], 


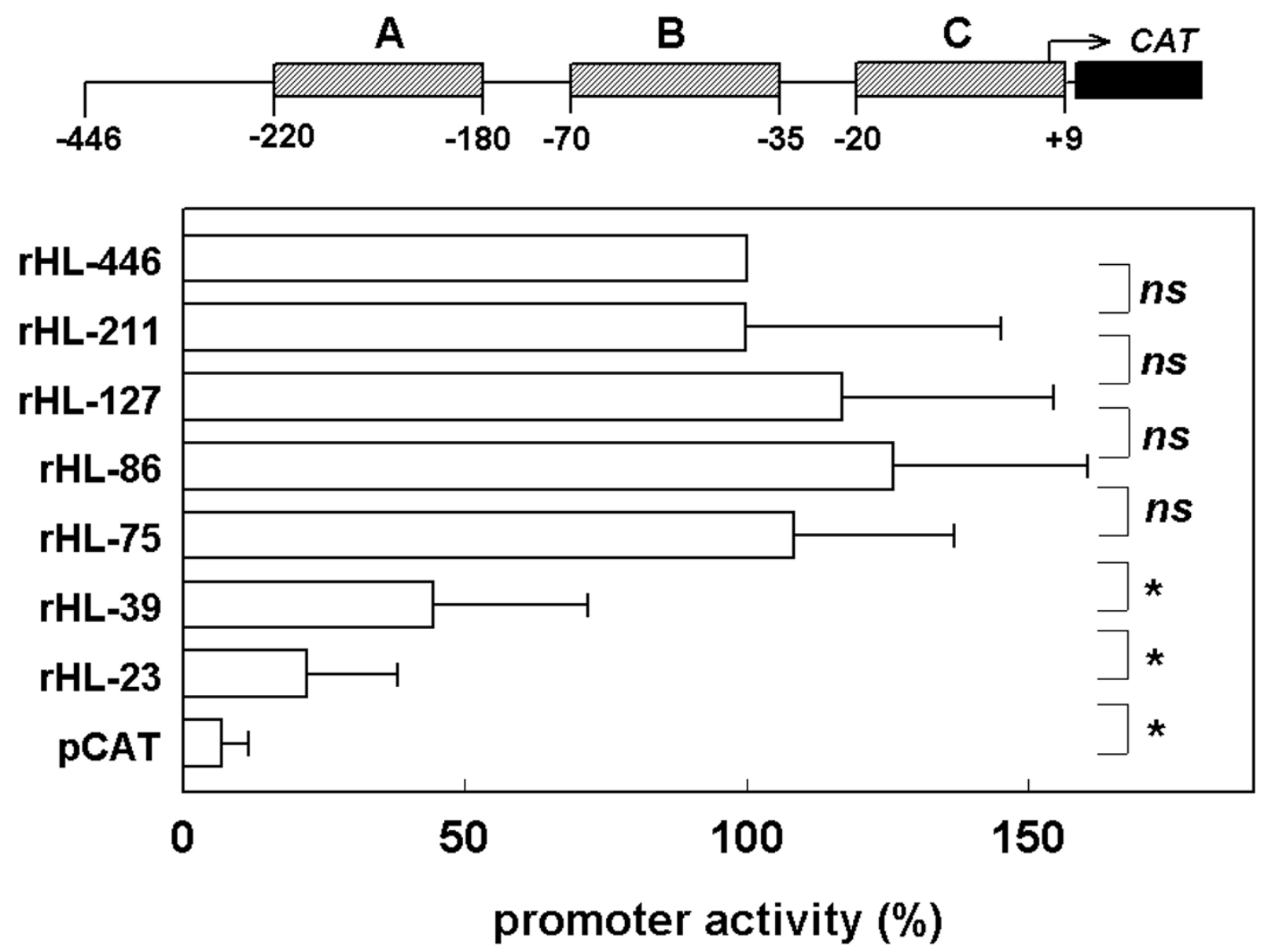

\section{Figure 5}

Effect of serial 5 '-deletions of the rat HL promoter region on transcriptional activity in HepG2 cells. Experiments were performed as described in the legends to figure 4. Data were expressed as percentage of the ratio measured in the rHL446 CAT - transfected cells, and are means \pm SD from $3-5$ independent experiments, each performed in triplicate. ${ }^{*}: P<0.05$; n.s.: not significant.

respectively. However, we missed an additional module (295 to -265) that has recently been identified as a functional DR4 site [25]. The cluster of TFBS within this module appeared to be conserved among human and mouse, but not among human and rat. Despite the relatively high homology between the mouse and rat over the proximal 5 '-flanking region of the HL gene (Table 1), the outcome of the genomic sequence analysis differed whether the rat or the mouse sequence was used. Hence, although searching genomic sequences for conserved clusters of TFBS is a valuable tool in predicting functionally important regulatory elements, this approach is sub optimal.

For two of the modules that are conserved among the four species, a significant contribution to basal transcription was confirmed by promoter-assays in HepG2 cells. For module $C(-25 /+5)$, this is not surprising since it contains the transcriptional start site itself, as well as a pyrimidinerich stretch that may serve as an initiator region (Inr). Module B (-80 to -40$)$ overlaps with a protected region in DNAse footprinting in rat liver [22] as well as in HepG2 cells [23], and contains a HNF1 binding site that has been implicated in liver-specific expression of the human HL gene by other groups [22-24]. Experimentally, we could not confirm a major role for module A $(-240$ to -200$)$ in determining basal transcription activity in HepG2 cells. This is surprising since it corresponds to a functional DR1 site [25], and perfectly matches with a protected region in DNAse footprinting in rat liver and human HepG2 nuclear extracts $[22,23]$, suggesting that this part of the HL promoter is occupied by transcription factors under basal conditions. Similarly, we could not confirm the role of the 


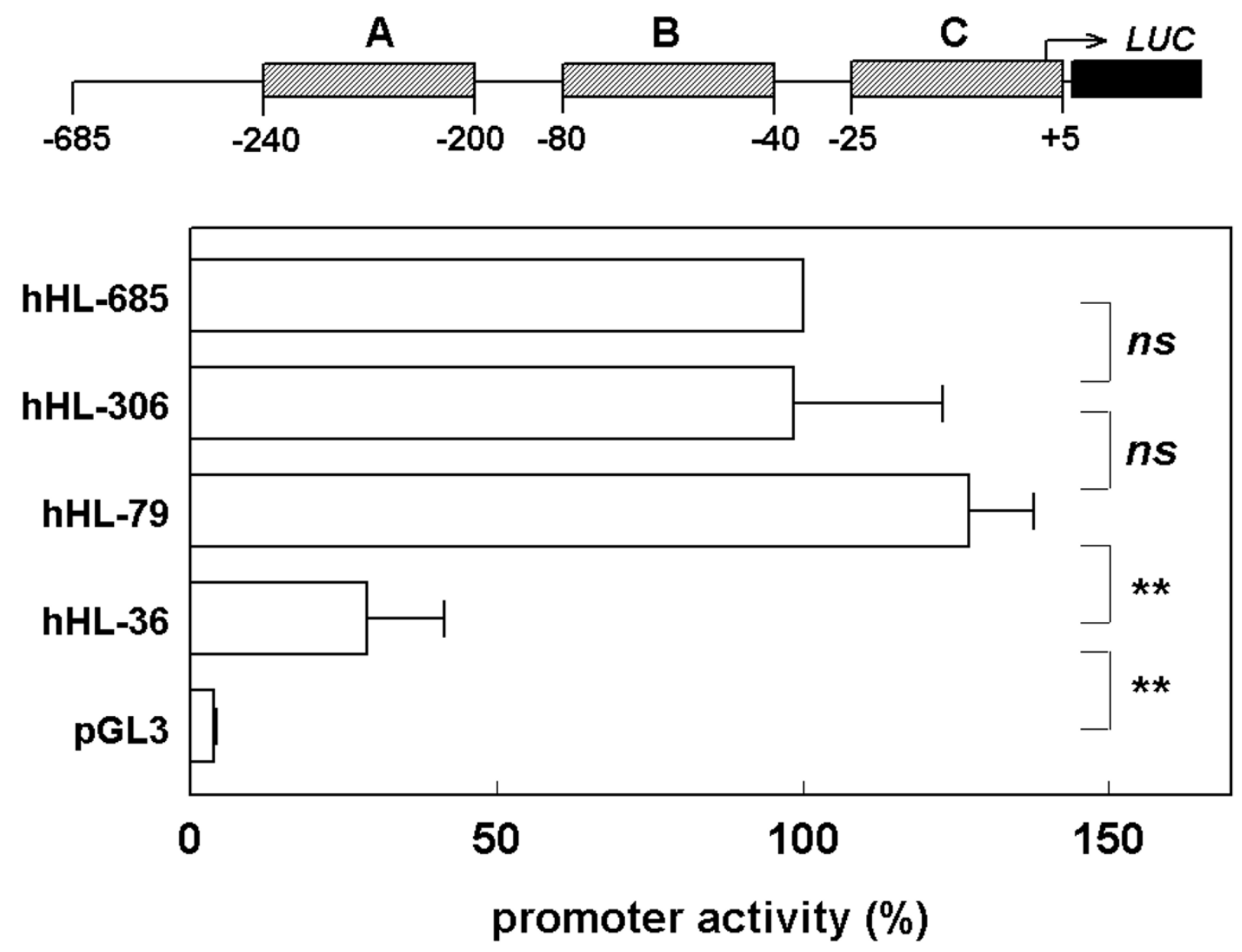

\section{Figure 6}

Effect of serial 5'-deletions of the human HL promoter region on transcriptional activity in HepG2 cells. HepG2 cells were transiently transfected with the indicated promoter-reporter constructs. At $48 \mathrm{~h}$ post-transfection, transcriptional activity was determined as the firefly over renilla luciferase activity. Data are expressed as percentage of the ratio measured in the hHL-685luc - transfected cells. Data are means \pm SD from 4 independent experiments, each performed in quadruplicate. *: $P<0.05$; n.s.: not significant.

DR4 module (-295 to -265) conserved among human and mouse, in basal transcriptional activity in HepG2 cells. We propose, therefore, that this part of the HL promoter region is involved in modulation of gene transcription under different hormonal or nutritional conditions.

We show here that the conserved module B (-80 to -40$)$ plays a dual role in mediating liver-restricted transcription of the HL gene. On the one hand, the module mediates moderate stimulation of minimal promoter activity in liver-derived HepG2 cells, and on the other hand, it mediates inhibition of minimal promoter activity in the nonhepatic HeLa cells. Of the potential TFBS identified in module $\mathrm{B}$, the liver-enriched HNF1 is a likely candidate for effecting the liver-specific activation of the HL pro- moter. Other groups have already suggested an important role for the HNF1 binding site [22-24], and in vitro HNF1 binding to this sequence has been demonstrated by gelshift assays [24]. Furthermore, HNF1 $\alpha$ knockout mice have 3.4 fold lower HL mRNA levels than control mice [28]. In primary hepatocytes, HL secretion increases with HNF1 $\alpha$ gene dosage [28]. However, HL mRNA and HL secretion are not completely lost by HNF1 $\alpha$ knockout, indicating that HNF1 $\alpha$ is not the only transcription factor determining HL expression in liver. HL secretion was only observed with hepatoma cell lines that express HNF1 $\alpha$ or HNF1 $\beta$ mRNA [24], but not all cell lines with detectable HNF $1 \alpha$ or $-\beta$ expression do also secrete HL. In fact, HL secretion correlated with expression of HNF4 rather than with HNF1 mRNA [24]. The HNF4 $\alpha$ gene itself is a target 


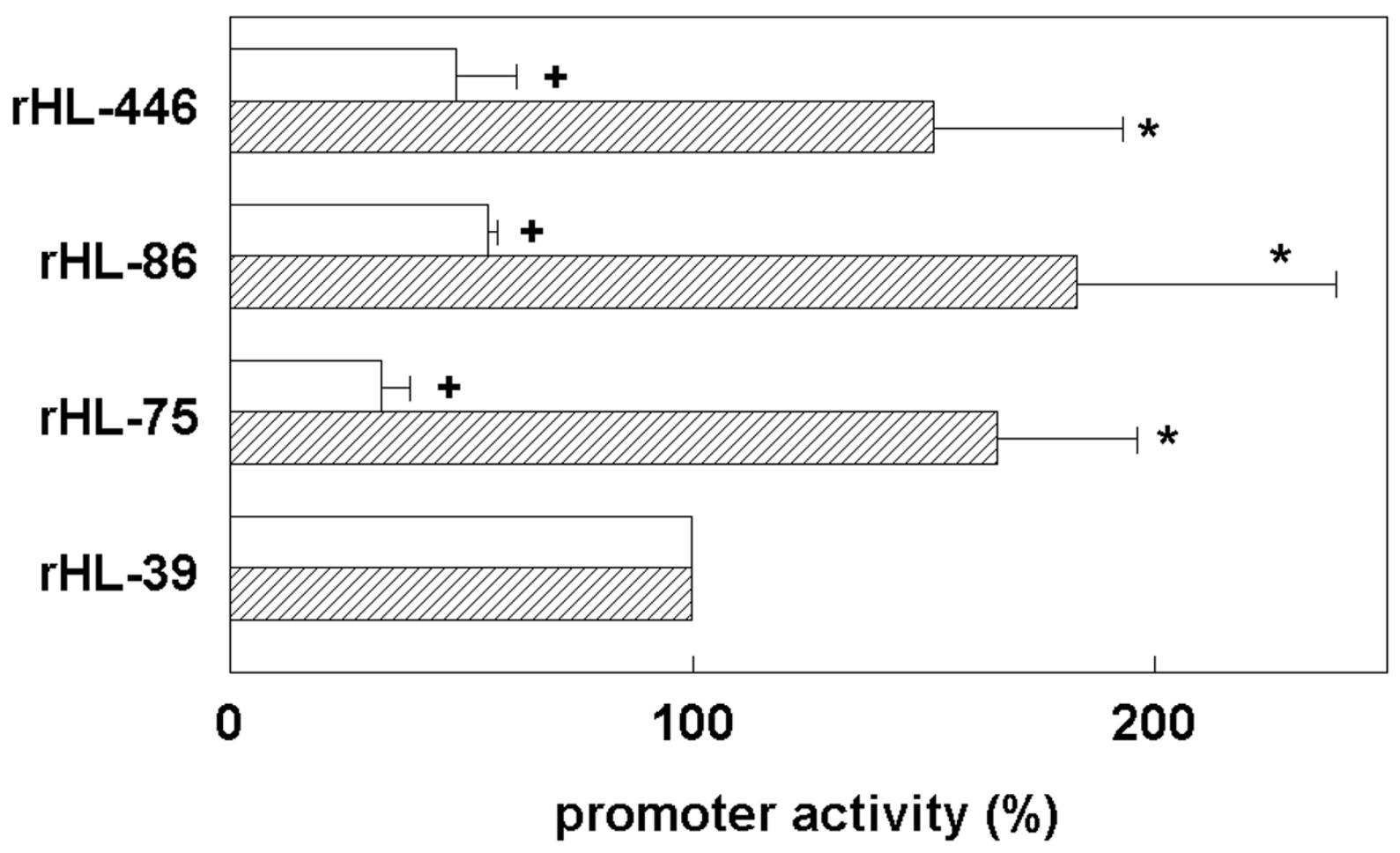

Figure 7

Transcriptional activity of the rat proximal HL promoter region in HepG2 and HeLa cells. HepG2 (hatched bars) and HeLa cells (open bars) were transiently transfected with the indicated promoter-reporter constructs. At $48 \mathrm{~h}$ post-transfection, transcriptional activity was determined as the ratio of CAT over $\beta$-galactosidase expression level. Data are expressed as percentage of the ratio measured in the rHL-39 CAT - transfected cells. Data are means \pm SD from 3 independent experiments, each performed in triplicate. ${ }^{*},+: P<0.05$ compared to $100 \%$ in HepG2 and HeLa cells, respectively.

of HNF1 $\alpha$ [29]. Since potential HNF4 $\alpha$ binding sites were detected in the conserved module A (as well as in the 295/-265 module), the liver-specific stimulation of HL promoter activity may well be mediated by $\mathrm{HNF} 4 \alpha$. In fact, $\mathrm{HNF} 4 \alpha$ is bound to the promoter regions of almost half of the actively transcribed genes in human liver [29] and therefore contributes to a large fraction of liver-specific gene expression. Sequence modules that contain both HNF1 and HNF4 binding sites are among the strongest predictors of liver-specific transcription [10]. Rufibach et al. [25] proposed that HNF1 $\alpha$ and HNF4 $\alpha$ independently and additively activate HL promoter activity. Which transcription factor(s) mediate inhibition of minimal pro- moter activity in cells of non-hepatic origin, remain(s) unknown at present.

\section{Conclusion}

In summary, we have shown here that a global multispecies comparison of non-coding sequences, followed by a search for conserved clusters of TFBS, has predicted the most important sequences involved in basal transcription of the HL gene. This in silico analysis does not identify all regulatory sequences in a particular gene, but enables the intelligent design of experiments towards identification of functional cis-regulatory elements and transactivating factors in gene regulation. This study illustrates the power of 
comparative genomics in the identification of TFBS that are functional in gene expression.

\section{Methods \\ Database analysis}

The annotated data of the mammalian genome sequence projects were accessed through the Ensembl genome server ( $e$ !42: Dec 2006) [30]. The exon-1 and 5'-upstream regulatory sequence of the hepatic lipase gene was available only for human (ENSG00000166035), rat (ENSRNOG00000015747),

(ENSMUSG00000032207),

(ENSETEG00000015177),

(ENSPTRG00000007115) and rhesus macaque (ENSMMUG00000009566). Multiple sequence alignment was performed with DNAMAN software package version 3.2 (Lynnon BioSoft, Quebec, Canada). Global sequence alignments were performed with the publicly available web-based tool mVista $[12,31]$ using the MLAGAN algorithm. A search for potential TFBS in the upstream regulatory region of a particular HL gene was performed online at Genomatix using the MatInspector program [2,32]. Clusters of TFBS that are conserved among the rat, mouse, human and macaque HL promoter regions were identified by the publicly available web-tool rVista $[11,12,31]$.

\section{Isolation of exon-I and the 5'-flanking region of the rat $H L$ gene}

A rat genomic library in $\lambda$ DASH II (Stratagene, La Jolla, CA, USA) was used for isolation of the HL promoter region, using a HL cDNA probe corresponding to exons-1 and -2 . The probe was generated by RT-PCR on $1 \mu \mathrm{g}$ rat liver RNA using the oligonucleotides (5'-GGT AAG ACG AGA GAC ATG G-3', nt 1-19; numbering according to [33]) and (5'-CCC GTG GAT GAT CAT GAC AA-3', nt 285-266) as forward and reverse primers, respectively. The RT-PCR product was isolated by agarose gel electrophoresis, and radiolabeled using $\left[\alpha^{32}-\mathrm{P}\right] \mathrm{dCTP}$ and the Megaprime kit from Amersham (Amersham, UK). Filters containing $10^{6}$ plaques were hybridized overnight at $42^{\circ} \mathrm{C}$ with 50 ng of the labeled cDNA probe in hybridization buffer (50\% (v/v) formamide, $0.5 \%(\mathrm{w} / \mathrm{v})$ SDS, 0.1 $\mathrm{mg} / \mathrm{ml}$ denaturated herring sperm DNA and $2 \times$ PIPES buffer; [34]). After washing in $0.2 \times$ sodium chloride/ sodium citrate $/ 0.5 \%$ SDS at $65^{\circ} \mathrm{C}$ for $5 \mathrm{~min}$, the filters were exposed to autoradiography film. Two positive clones were identified, which were plaque-purified three times. One of these clones was selected for further analysis. Phage DNA was isolated and digested with EcoRI. A 6 kb fragment [35] was subcloned into pBluescript KS(pBsE6) and its identity with the 5'-regulatory region of the rat HL gene was verified by sequence analysis.

\section{Construction of reporter plasmids}

The clone in pBluescript containing the $6 \mathrm{~kb} E c o R I$ fragment of the rat HL gene (pBsE6) was used to generate promoter-reporter constructs in pCAT-Basic (Promega, Madison, WI, USA). By digestion with PstI and XbaI, a 1.85-kb PstI/PstI, a 0.32-kb PstI/XbaI and a 0.15-kb XbaI/

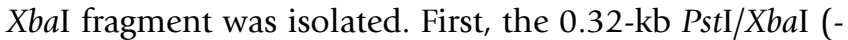
446/-127; numbering according to [35]) fragment was cloned into pCAT-Basic. From this construct, the rHL-446 CAT plasmid was generated by insertion of the $0.15-\mathrm{kb}$ XbaI $(-127 /+9)$ fragment. Subsequently, rHL-2287 CAT was generated by insertion of the 1.85-kb PstI (-2287/446) fragment into rHL-446. The rHL-127 CAT construct was made by subcloning the $0.15-\mathrm{kb} \mathrm{XbaI}(-127 /+9)$ fragment into pCAT-Basic.

From the rHL-2287 CAT vector, the 5'-truncated rHL1697, rHL-1041 and rHL-747 constructs were generated by PCR using HindIII-restriction site-containing oligonucleotides $3 \mathrm{~F}, 4 \mathrm{~F}$ and $5 \mathrm{~F}$ as upstream primer, respectively, and the CAT-gene specific oligonucleotide CATrev2 as downstream-primer (Table 2). After digestion of the PCR products with HindIII and PstI, the DNA fragments were purified by electrophoresis through agarose gel, and subsequently ligated into the rHL-446 CAT plasmid that had been digested with the same restriction enzymes. Similarly, the rHL-211 construct was generated from the rHL446 CAT by PCR using oligonucleotides $9 \mathrm{~F}$ and CATrev2 as upstream and downstream primer, respectively, followed by ligation into the HindIII and $\mathrm{XbaI}$ sites of rHL446 CAT. Finally, the rHL-75, rHL-39 and rHL-23 constructs were generated from pBsE6 using $7 \mathrm{~F}, 6 \mathrm{~F}$ and $11 \mathrm{~F}$ as upstream, and T3Primer as downstream primer, respectively, followed by digestion and ligation into the HindIII and PstI sites of pCAT-Basic; subsequently, the resulting plasmids were digested with $\mathrm{XbaI}$ followed by self-ligation.

Human HL promoter constructs were made in the pGL3Basic luciferase reporter plasmid (Promega, Madison, WI, USA), starting from the hHL(-685/+13)-CAT plasmid described previously [36]. An upstream SacI restriction site was introduced by PCR using the HHL-685Sac primer (Table 2) and the downstream HHL+13Xba primer. After digestion with $S a c I$ and $X b a I$, the gel-purified DNA products were ligated into the $S a c I$ and NheI sites of pGL3, thus generating the hHL-685Luc plasmid. Similarly, hHL306Luc, hHL-79Luc and hHL-36Luc plasmids were generated by using HHL-306Nhe, HHL-79Kpn, and HHL$36 \mathrm{Kpn}$ as upstream primers, respectively.

To test the enhancer activity of conserved upstream sequences, the $-14 \mathrm{~kb},-22 \mathrm{~kb}$ and $-10 \mathrm{~kb}$ elements were inserted into the enhancer site of the hHL-685Luc plasmid using the BamHI and SalI restriction sites. Human 
Table 2: Oligonucleotides used to generate serial 5'-deletions of the rat and human HL promoter region.

\begin{tabular}{|c|c|c|c|c|}
\hline \multirow{2}{*}{$\begin{array}{l}\text { Name } \\
3 \mathrm{~F}\end{array}$} & \multicolumn{2}{|c|}{ species and orientation $^{\mathrm{a}}$} & \multirow{2}{*}{$\begin{array}{c}\text { position }^{b} \\
-1697 /-1675\end{array}$} & \multirow{2}{*}{$\begin{array}{l}5^{\prime} \rightarrow 3^{\prime} \text { sequence }^{c} \\
\text { cggaagc TTA GCA GAC AGC GAT TGG C }\end{array}$} \\
\hline & r & $\mathrm{F}$ & & \\
\hline $4 \mathrm{~F}$ & $r$ & $\mathrm{~F}$ & $-1048 /-1030$ & cggaag CTT GCC TCC TCC TGA GTG C \\
\hline $5 \mathrm{~F}$ & $r$ & $\mathrm{~F}$ & $-754 /-736$ & cggaagc TTG TCC AGG GCG TCC ATA C \\
\hline $9 \mathrm{~F}$ & $r$ & $\mathrm{~F}$ & $-211 /-196$ & cggaagctt AGC TTG GCT CAA AAG G \\
\hline $8 \mathrm{~F}$ & $r$ & $\mathrm{~F}$ & $-86 /-7 \mid$ & cggaagctt GTG TTC AAA TAC TGG G \\
\hline $7 F$ & $r$ & $\mathrm{~F}$ & $-75 /-58$ & cggaagct TGG GTA ACA TGT TTT AGG \\
\hline $6 \mathrm{~F}$ & $r$ & $\mathrm{~F}$ & $-39 /-22$ & cggaag CTT CCA CAA CTA AAT ACC \\
\hline $\mathrm{IIF}$ & $r$ & $\mathrm{~F}$ & $-23 /-8$ & cggaagctt CCA AGA AGC ATT CTG G \\
\hline HHL-685Sac & $\mathrm{h}$ & $\mathrm{F}$ & $-685 /-667$ & ccgagctc TGG TCG CCT TTT CCC TAC C \\
\hline HHL-306Nhe & $\mathrm{h}$ & $\mathrm{F}$ & $-306 /-291$ & gcatgctagc GAA GCC ACC TAC CCC G \\
\hline HHL-79Kpn & $\mathrm{h}$ & $\mathrm{F}$ & $-79 /-55$ & ggggtacc TAA CAT GTT GAG AGG \\
\hline HHL-36Kpn & $\mathrm{h}$ & $\mathrm{F}$ & $-36 /-20$ & ggggtac CAA AGT ATC TAA TAG GC \\
\hline $\mathrm{HHL}+13 \mathrm{Xba}$ & $\mathrm{h}$ & $\mathrm{R}$ & $+13 /-6$ & gctctaga CTT GGT AAT TTC TGA AGC C \\
\hline HHL-IOKfW & $\mathrm{h}$ & $\mathrm{F}$ & $-10457 /-10437$ & gtcgac GAA GGA TCA GGT GAG GGA TGG \\
\hline HHL-IOKrev & $\mathrm{h}$ & $\mathrm{R}$ & $-9956 /-9975$ & ggatcc GCT TCA AGG GCA ATG AAA GC \\
\hline HHL-I 4Kfw & $\mathrm{h}$ & $\mathrm{F}$ & $-|4202 /-| 4 \mid 83$ & gtcgac GAC TTG GGG ATA TCC ACA TC \\
\hline HHL- I $4 \mathrm{Krev}$ & $\mathrm{h}$ & $\mathrm{R}$ & $-13524 /-13549$ & ggatc CTG GTA AAA GGA CAT GAA CAA TAT GG \\
\hline HHL-22Kfw & $\mathrm{h}$ & $\mathrm{F}$ & $-21929 /-21909$ & gtcgac GAT GGG AAA TGG ACC TAC AGC \\
\hline HHL-22Krev & $\mathrm{h}$ & $\mathrm{R}$ & $-21543 /-21562$ & ggatcc GAT GAG GAC TGA TTC TCA GC \\
\hline CATREV $^{2 d}$ & - & $\mathrm{R}$ & & gca act gac tga aat gcc tc \\
\hline T3primere & - & $\mathrm{R}$ & & att aac cct cac taa ag \\
\hline
\end{tabular}

$a: r$ and $h$ : rat and human, respectively; $F$ and $R$ : forward and reverse orientation.

b: numbering of the rat and human sequence according to [35] and Ensembl e!42 [26], respectively.

c: rat and human $\mathrm{HL}$ specific parts of the primer sequences are given in capitals.

d: oligonucleotide specific for PCAT-Basic.

e: oligonucleotide specific for pBluescript.

genomic DNA was isolated from a buffy coat, and the -14 $\mathrm{kb},-22 \mathrm{~kb}$ and $-10 \mathrm{~kb}$ elements were PCR amplified using specific primers (Table 2). The PCR products were cloned into the pGEM T-easy vector (ProMega, Madison, WI, USA). After digestion of the plasmids with BamHI and SalI, the inserts were cloned into hHL-685Luc in either sense or anti-sense orientation.

All clones were verified by DNA sequencing using the Thermo-sequenase dye terminator kit (Amersham, UK) and the ABI 377 sequencer (Applied Biosystems, Foster City, CA, USA).

\section{Promoter reporter assays}

HepG2 hepatoma cells and HeLa cells were cultured at $37^{\circ} \mathrm{C}$ and $5 \% \mathrm{CO}_{2}$ in Dulbecco's modified Eagle's medium (ICN, Costa Mesa, CA, USA) supplemented with $10 \%(\mathrm{v} / \mathrm{v})$ fetal calf serum (Gibco, Breda, Netherlands) and penicillin/streptomycin. Transfection of HepG2 cells with CAT-reporter constructs was performed by the calcium-phosphate co-precipitation method. At $24 \mathrm{~h}$ before transfection, the cells were plated in 6-wells plates at 20$30 \%$ confluence. At $3 \mathrm{~h}$ before transfection, the medium was refreshed. Cells were co-transfected with $2.5 \mu \mathrm{g} /$ well of the CAT reporter test plasmid and $0.2 \mu \mathrm{g} /$ well of control RSV $\beta$-galactosidase expression plasmid (Promega) [36]. Parallel transfections with SV40-CAT-Control and empty
pCAT-Basic plasmids were used as controls. Fourty-eight hours post-transfection, cell lysates were prepared. CAT and $\beta$-galactosidase were determined by ELISA (Roche). Promoter activity was expressed as pg CAT/ng $\beta$-galactosidase to correct for differences in cell number and transfection efficiency.

Transfections of HepG2 and HeLa cells with the luciferase-reporter constructs were performed in 24-wells plates with Lipofectamine Plus (Invitrogen, Groningen, Netherlands) using $0.4 \mu \mathrm{g}$ of the luciferase-reporter construct and $20 \mathrm{ng}$ of pRL-CMV (Promega) per well [37]. Cell extracts were prepared at $48 \mathrm{~h}$ post-transfection. The luciferase activity in the cell extracts was determined with the FireLight kit (Perkin-Elmer, Boston MA, USA) and the Packard Top Count NXT luminometer. Data were normalized for the Renilla activity measured in the same sample.

\section{Statistics}

Experimental data are expressed as mean \pm SD. Differences were tested for statistical significance by paired Student's $t$-test.

\section{List of abbreviations}

CAT, chloramphenicol acetyltransferase; HDL, high-density lipoprotein; HL, hepatic lipase; HNF, hepatocyte 
nuclear factor; kb, kilo basepairs; TFBS, transcription factor binding sites

\section{Authors' contributions}

DvD and GJB carried out the biochemical assays. HJ and AJMV conceived of the study and participated in its design and coordination. AJMV carried out the comparative genomic analysis, and drafted the manuscript. All authors read and approved the final manuscript.

\section{Acknowledgements}

We thank M. Heuveling, M. van Leeuwen and R. Voorzaat for expert technical assistance, and B. Hogema and J.M.J. Lamers for critically reading the manuscript. Part of this study was supported by grants 94.064 and $200 I B I 74$ from the Dutch Heart Foundation.

\section{References}

I. Wingender E, Chen X, Fricke E, Geffers R, Hehl R, Liebich I, Krull M, Matys V, Michael H, Ohnhauser R, Pruss M, Schacherer F, Thiele S, Urbach S: The TRANSFAC system on gene expression regulation. Nucleic Acids Res 200I, 29:28I-283.

2. Quandt K, Frech K, Karas H, Wingender E, Werner T: MatInd and MatInspector: new fast and versatile tools for detection of consensus matches in nucleotide sequence data. Nucleic Acids Res 1995, 23:4878-4884.

3. Fickett JW, Wasserman WW: Discovery and modeling of transcriptional regulatory regions. Curr Opin Biotechnol 2000, II:19-24.

4. Zhang Z, Gerstein M: Of mice and men: phylogenetic footprinting aids the discovery of regulatory elements. J Biol 2003, 2: I I.

5. Hardison RC: Conserved noncoding sequences are reliable guides to regulatory elements. Trends Genet 2000, 16:369-372.

6. Levy S, Hannenhalli S, Workman C: Enrichment of regulatory signals in conserved non-coding genomic sequence. Bioinformatics 200I, 17:87I-877.

7. Cora D, Herrmann C, Dieterich C, Di Cunto F, Provero P, Caselle M: Ab initio identification of putative human transcription factor binding sites by comparative genomics. $B M C$ Bioinformatics 2005, 6: 110 .

8. Pennacchio LA, Ahituv N, Moses AM, Prabhakar S, Nobrega MA, Shoukry M, Minovitsky S, Dubchak I, Holt A, Lewis KD, Plajzer-Frick I, Akiyama J, De Val S, Afzal V, Black BL, Couronne O, Eisen MB, Visel $A$, Rubin EM: In vivo enhancer analysis of human conserved non-coding sequences. Nature 2006, 444:499-502.

9. Wagner A: Genes regulated cooperatively by one or more transcription factors and their identification in whole eukaryotic genomes. Bioinformatics 1999, 15:776-784.

10. Krivan W, Wasserman WW: A predictive model for regulatory sequences directing liver-specific transcription. Genome Res 200I, I I:I559-I566.

II. Loots GG, Ovcharenko I, Pachter L, Dubchak I, Rubin EM: rVista for comparative sequence-based discovery of functional transcription factor binding sites. Genome Res 2002, I 2:832-839.

12. Frazer KA, Pachter L, Poliakov A, Rubin EM, Dubchak I: VISTA: computational tools for comparative genomics. Nucleic Acids Res 2004, 32:W273-9.

13. Doolittle MH, Wong H, Davis RC, Schotz MC: Synthesis of hepatic lipase in liver and extrahepatic tissues. J Lipid Res 1987, 28:1326-1334.

14. Semenkovich CF, Chen SH, Wims M, Luo CC, Li WH, Chan L: Lipoprotein lipase and hepatic lipase mRNA tissue specific expression, developmental regulation, and evolution. J Lipid Res 1989, 30:423-431.

15. Perret B, Mabile L, Martinez L, Terce F, Barbaras R, Collet X: Hepatic lipase: structure/function relationship, synthesis, and regulation. J Lipid Res 2002, 43: I I63-II69.

16. Schultz CJ, Blanchette-Mackie EJ, Scow RO: Adrenal and liver in normal and cld/cld mice synthesize and secrete hepatic lipase, but the lipase is inactive in cld/cld mice. J Lipid Res 2000, 41:214-225.
17. Gonzalez-Navarro H, Nong Z, Freeman L, Bensadoun A, Peterson K, Santamarina-Fojo S: Identification of mouse and human macrophages as a site of synthesis of hepatic lipase. J Lipid Res 2002, 43:67I-675.

18. Jansen H, De Greef WJ: Heparin-releasable lipase activity of rat adrenals, ovaries and testes. Biochem J 198I, 196:739-745.

19. Hixenbaugh EA, Sullivan TR Jr., Strauss JF 3rd, Laposata EA, Komaromy M, Paavola LG: Hepatic lipase in the rat ovary. Ovaries cannot synthesize hepatic lipase but accumulate it from the circulation. J Biol Chem 1989, 264:4222-4230.

20. Galan X, Peinado-Onsurbe J, Julve J, Ricart-Jane D, Robert MQ, Llobera M, Ramirez I: Inactive hepatic lipase in rat plasma. J Lipid Res 2003, 44:2250-2256.

21. Jansen H, Verhoeven AJ, Sijbrands EJ: Hepatic lipase: a pro- or anti-atherogenic protein? J Lipid Res 2002, 43:1352-1362.

22. Hadzopoulou-Cladaras M, Cardot P: Identification of a cis-acting negative DNA element which modulates human hepatic triglyceride lipase gene expression. Biochemistry 1993, 32:9657-9667.

23. Oka K, Ishimura-Oka K, Chu MJ, Chan L: Transcription of the human hepatic lipase gene is modulated by multiple negative elements in HepG2 cells. Gene 1996, 180:69-80.

24. Chang SF, Scharf JG, Will H: Structural and functional analysis of the promoter of the hepatic lipase gene. Eur J Biochem I997, 247:148-159.

25. Rufibach LE, Duncan SA, Battle M, Deeb SS: Transcriptional regulation of the human hepatic lipase (LIPC) gene promoter. J Lipid Res 2006, 47: I463-1477.

26. Hubbard TJ, Aken BL, Beal K, Ballester B, Caccamo M, Chen Y, Clarke L, Coates G, Cunningham F, Cutts T, Down T, Dyer SC, Fitzgerald S, Fernandez-Banet J, Graf S, Haider S, Hammond M, Herrero J, Holland R, Howe K, Johnson N, Kahari A, Keefe D, Kokocinski F, Kulesha E, Lawson D, Longden I, Melsopp C, Megy K, Meidl P, Ouverdin B, Parker A, Prlic A, Rice S, Rios D, Schuster M, Sealy I, Severin J, Slater G, Smedley D, Spudich G, Trevanion S, Vilella A, Vogel J, White S, Wood M, Cox T, Curwen V, Durbin R, Fernandez-Suarez XM, Flicek P, Kasprzyk A, Proctor G, Searle S, Smith J, Ureta-Vidal A, Birney E: Ensembl 2007. Nucleic Acids Res 2007, 35:D610-7.

27. Prabhakar S, Poulin F, Shoukry M, Afzal V, Rubin EM, Couronne O, Pennacchio LA: Close sequence comparisons are sufficient to identify human cis-regulatory elements. Genome Res 2006, 16:855-863.

28. Shih DQ, Bussen M, Sehayek E, Ananthanarayanan M, Shneider BL, Suchy FJ, Shefer S, Bollileni JS, Gonzalez FJ, Breslow JL, Stoffel M: Hepatocyte nuclear factor- I alpha is an essential regulator of bile acid and plasma cholesterol metabolism. Nat Genet 200I, 27:375-382.

29. Odom DT, Zizlsperger N, Gordon DB, Bell GW, Rinaldi NJ, Murray HL, Volkert TL, Schreiber J, Rolfe PA, Gifford DK, Fraenkel E, Bell GI, Young RA: Control of pancreas and liver gene expression by HNF transcription factors. Science 2004, 303:|378-|38|.

30. Ensembl [http://www.ensembl.org]

31. Vista [http://genome.lbl.gov/vista]

32. Genomatix Software GmbH [http://www.genomatix.de]

33. Komaromy MC, Schotz MC: Cloning of rat hepatic lipase cDNA: evidence for a lipase gene family. Proc Natl Acad Sci U S A 1987, 84: $1526-1530$.

34. Sambrook J FEF Maniatis T: Molecular Cloning: A Laboratory Manual. Cold Spring Harbor, Cold Spring Harbor Press; 1989.

35. Sensel MG, Legrand-Lorans A, Wang ME, Bensadoun A: Isolation and characterization of clones for the rat hepatic lipase gene upstream regulatory region. Biochim Biophys Acta 1990, 1048:297-302.

36. Botma G], Verhoeven AJ, Jansen $H$ : Hepatic lipase promoter activity is reduced by the C-480T and G-216A substitutions present in the common LIPC gene variant, and is increased by Upstream Stimulatory Factor. Atherosclerosis 200I, 154:625-632.

37. Botma GJ, van Deursen D, Vieira D, van Hoek M, Jansen $H$, Verhoeven AJ: Sterol-regulatory-element binding protein inhibits upstream stimulatory factor-stimulated hepatic lipase gene expression. Atherosclerosis 2005, 179:61-67.

38. Warren RJ, Ebert DL, Mitchell A, Barter PJ: Rabbit hepatic lipase cDNA sequence: low activity is associated with low messenger RNA levels. J Lipid Res 1991, 32:1333-1339. 